\section{A MICROSTRIP-COUPLED SLOT-LOOP ANTENNA FOR INTEGRATED RECEIVERS IN THE MILLIMETER-WAVE BAND}

Pablo Otero, ${ }^{1}$ Alejandro Álvarez-Melcón, ${ }^{1}$

Jean-François Zürcher, ${ }^{1}$ and Juan R. Mosig ${ }^{1}$

'Laboratoire d'Électromagnétisme et d'Acoustique (LEMA)

École Polytechnique Fédérale de Lausanne (EPFL)

$\mathrm{CH}-1015$ Lausanne, Switzerland

Received 3 December 1997

ABSTRACT: A square-loop slot antenna, printed at the back surface of an extended hemispherical lens, is examined as a candidate for millimeter-wave (mm-wave) integrated-circuit Schottky-diode mixers. The loop slot is etched in a microwave substrate, and coupled to a microstrip line section printed on the other side of the substrate. A Schottky barrier diode shunted to ground is the mixing device. The input impedance of the antenna has been optimized to conjugate match the Schottky diode. To design and optimize the antenna, an efficient full-wave method of moments (MoM) technique, which solves the mixed potential integral equation (MPIE) in the stratified medium, has been developed. Patterns have been computed using the physical optics approximation and diffraction theory. The proposed configuration shows very wideband performance. Simulation results compare well with measurements. The proposed configuration is suitable to be scaled down for operation in the submillimeter-wave (submm-wave) band. (c) 1998 John Wiley \& Sons, Inc. Microwave Opt Technol Lett 18: 91-95, 1998.

Key words: mixed potential integral equation (MPIE); method of moments (MoM); planar antennas; slot antennas; millimeter wave

\section{INTRODUCTION}

An increased interest in planar integrated receivers for the $\mathrm{mm}$ - and submm-wave bands has been observed recently. The main reason is the desire to replace the waveguide-based technology currently used in spaceborne applications. In this context, previous work has shown the feasibility of using uniplanar technology (coplanar transmission-line-fed slot antennas), coupled to extended hemispherical dielectric lenses, and integrating a mixing device [1]. This design is sought to operate as a front-end feed for reflector-type antennas. However, when scaled to the submm-wave band, the location of the mixing device, a Schottky barrier diode, integrated with the coplanar waveguide, results in an asymmetric configuration with too high sidelobe levels [2]. To ensure the symmetry and to isolate the diode from the radiation semispace, an antenna configuration, already used in the 1980s in $C$ - and $X$-bands, has been tried [3]. Figure 1, where all units are millimeters, shows the proposed design. The Schottky diode is only symbolically represented, as well as the intermediate frequency (IF) filter and the extended hemispherical lens. The IF filter is a nine-section, quarter-wavelength transformer, low-pass filter with a cutoff frequency of about $40 \mathrm{GHz}$. The low relative permittivity substrate is mounted on the plane surface of the dielectric lens. The radius of the lens is much larger than the one shown in Figure 1. The utilized substrate is RT/Duroid 5870, $\epsilon_{r}=2.33, \tan \delta=$ 0.0012 , and $h=0.254 \mathrm{~mm}$ [4]. The face which is in contact with the lens has a conductor plane where a square slot loop has been etched. On the other substrate's face, a microstrip line section couples to the slot loop at one end and feeds the mixing diode at the other. The IF signal is extracted from the same diode terminal, as shown in Figure 1. For our experi- ments, the local oscillator (LO) signal has been injected through the same filter, so that subharmonic mixing has been performed. For a possible scaled prototype operating at submm-wave frequencies, quasioptical LO signal injection can also be used.

The two main problems of the original design, when such high frequencies are used, are the appearance of surface waves and the too high losses in microstrip lines. The former is controlled by an appropriate choice of the substrate thickness and permittivity. As shown by Gupta et al. [5], the cutoff frequency $f_{c}$ of the first higher order mode in a microstrip line is given approximately by

$$
f_{c} \simeq \frac{300}{\sqrt{\epsilon_{r}}(2 W+0.8 h)}
$$

where $f_{c}$ is in gigahertz, $\epsilon_{r}$ is the substrate relative permittivity, $W$ is the microstrip width in millimeters, and $h$ is the substrate thickness, also in millimeters. For the RT/Duroid 5870 substrate used in the present design, to have $f_{\text {op }}<f_{c}$, where $f_{\text {op }}$ is the operating frequency, we need

$$
W(\mathrm{~mm})<\frac{98}{f_{\text {op }}(\mathrm{GHz})}-0.1 .
$$

The widths of the designed microstrip lines satisfy this condition. Equation (1) shows that the smaller $\epsilon_{r}$ and $h$ are, the less power conveyed into surface waves. This is a design advantage because the larger the effective relative permittivity ratio at both sides of the slot loop plane, the larger the front-to-back power ratio of the antenna [6].

The second problem of the design is due to the high ohmic losses of microstrip lines at $\mathrm{mm}$ - and submm-wave frequencies. The present solution uses the shortest possible length of microstrip at these frequencies, as can be seen in Figure 1. Beyond the diode, only the IF signal propagates. A very important feature of the design is that the slot loop perimeter and the microstrip length are quite independent. This fact has two main advantages: first, diode matching can be carried out with little concern about the slot loop size and, second, the frequency band can be widened compared to previous uniplanar designs [7]. Indeed, it is possible that the antenna itself conjugate matches the diode. This technique has already been used with success in the previously mentioned designs. The sought input impedance of the antenna is therefore not $50 \Omega$, but rather $Z_{\text {diode }}^{*}$. A typical Schottky diode impedance at $65 \mathrm{GHz}, Z_{\text {diode }}=(50-j 50) \Omega$, has been used.

\section{ANALYTIC METHODS}

2.1. Theoretical Model. For the analysis of the radiating structure proposed, a standard integral equation formulated in the space domain has been derived. In the slot interfaces, Love's equivalence principle is used, and suitable magnetic currents are defined to help impose the continuity conditions for the transverse electric and magnetic fields. The self-interactions (electric-electric and magnetic-magnetic) are solved with the aid of the vector and scalar potential Green's functions, especially well suited due to their weak singular behaviors when the source-observer distance $\rho$ vanishes [8]. All other interactions, nonexhibiting singularities, are treated directly using the electric and magnetic field Green's func- 


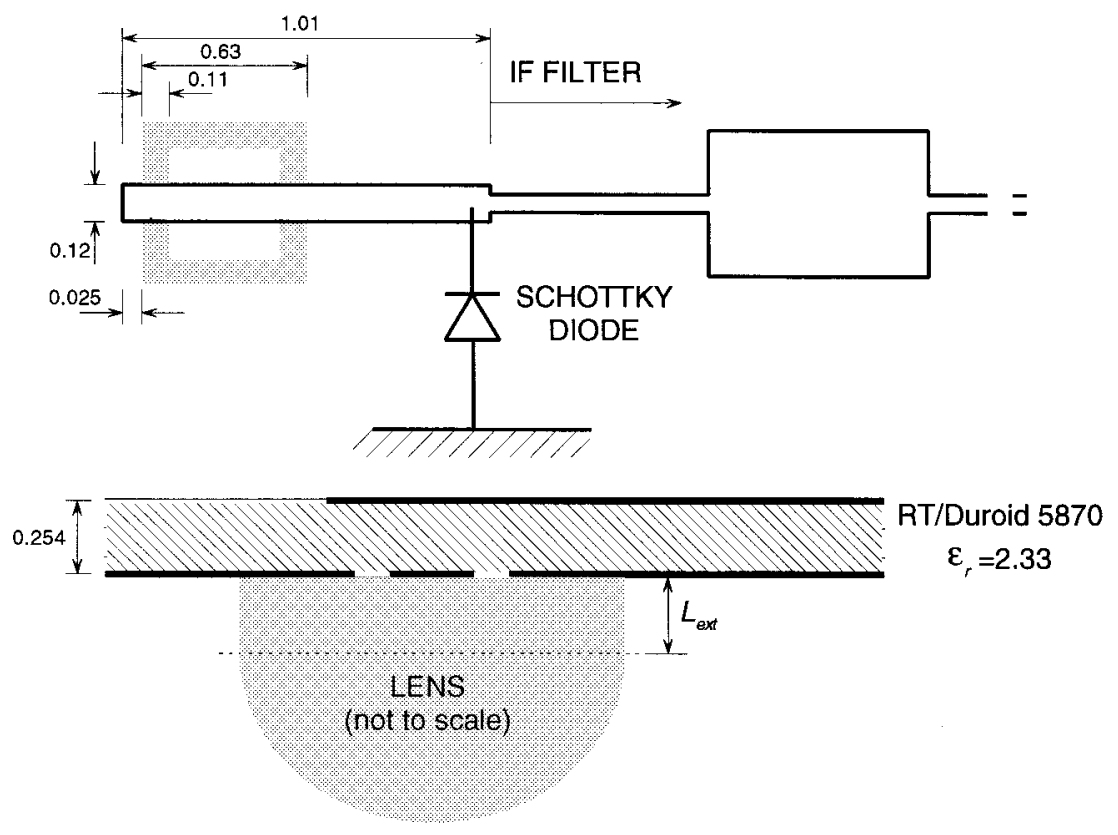

Figure 1 Proposed design. All units in millimeters

tions, thus leading to a very compact analytical treatment of the problem.

Multilayered media potential and field Green's functions are first obtained in the spectral domain using the transverse equivalent transmission line network representation of the structure along the stratified axis. Following this formalism, the spectral-domain Green's functions associated to electric and magnetic dipoles are easily obtained by computing equivalent voltages and currents in the resulting transmission line networks [9]. Once the spectral-domain Green's functions are computed, their spatial-domain counterparts can be obtained through the well-known Sommerfeld integral, and the efficient numerical techniques described in [8] have been used. After efficient evaluation of the space-domain Green's functions, the imposition of the boundary conditions for the fields in the specific structure shown in Figure 1 yields the following vector coupled system of integral equations on the unknown induced electric $\left(\bar{J}_{s}\right)$ and magnetic $\left(\bar{M}_{s}\right)$ currents:

$$
\begin{aligned}
& \bar{E}^{(e)}=j \omega \iint_{S_{e}} \overline{\bar{G}}_{A} \cdot \bar{J}_{s} d S^{\prime}-\frac{1}{j \omega} \iint_{S_{e}} \nabla G_{V} \nabla^{\prime} \cdot \bar{J}_{s} d S^{\prime} \\
& -\iint_{S_{m}} \overline{\bar{G}}_{E M} \cdot \bar{M}_{s} d S^{\prime} \\
& 0=j \omega \iint_{S_{m}}\left(\overline{\bar{G}}_{F u}+\overline{\bar{G}}_{F l}\right) \cdot \bar{M}_{s} d S^{\prime} \\
& -\frac{1}{j \omega} \iint_{S_{m}} \nabla\left(G_{W u}+G_{W l}\right) \nabla^{\prime} \cdot \bar{M}_{s} d S^{\prime} \\
& -\iint_{S_{e}} \overline{\bar{G}}_{H J} \cdot \bar{J}_{s} d S^{\prime}
\end{aligned}
$$

where $\bar{E}^{(e)}$ is the exciting or impressed field due to the generator applied to the strip line, $S_{e}$ is the surface of the strip line, and $S_{m}$ is the surface of the slot loop. The subscripts $u$ and $l$ of Green's functions stand for above and below, respectively, the conductor plane where the slots are etched.
2.2. Numerical Methods. For the solution of the vector-coupled system of integral equations previously derived, a Galerkin method of moments (MoM) algorithm based on subsectional rooftop functions has been developed. The main difficulty in the implementation of the proposed approach is the efficient numerical evaluation of the fourfold overlapping integrals between the spatial-domain Green's functions and the rooftop basis and test functions. In the present work, the cross-correlation integrals of the rooftop functions have been used together with the symmetry properties of Green's functions to systematically reduce all fourfold integrals into double integrals, thus considerably reducing the computational effort. For the singular cases, a further transformation to the polar plane is performed in order to analytically extract the $(1 / \rho)$ singular behavior of the potential Green's functions. Thanks to the analytical extraction procedure applied, the remainder function behaves very smoothly, and standard numerical integration techniques can be applied, obtaining good accuracy with a limited number of integration points. In Figure 2, we present the value obtained for a typical singular MoM element as a function of the number of integration points. The computation is performed for the structure shown in Figure 1 and for the frequency of $70 \mathrm{GHz}$. We can observe that good convergence is obtained with only $(4 \times 4)$ integration points, the relative error being smaller than $0.1 \%$.

The developed software tool takes only $4 \mathrm{~s}$ per frequency point on an HP712/80 platform to complete the analysis of the antenna structure shown in Figure 1, when only one cell is used in the transverse direction of the strip line and loop. Even when, looking for increased accuracy, the discretization is performed with three cells in the transverse direction, the software still only takes $18 \mathrm{~s}$ per frequency point on the same platform. These results clearly indicate that the developed software can be used as a real tool for the efficient optimization of this type of antennas.

2.3. Lens Analysis. The field in the far region from the lens may be calculated by means of the Love's equivalence principle, which allows substitution of the lens by a perfect conduc- 


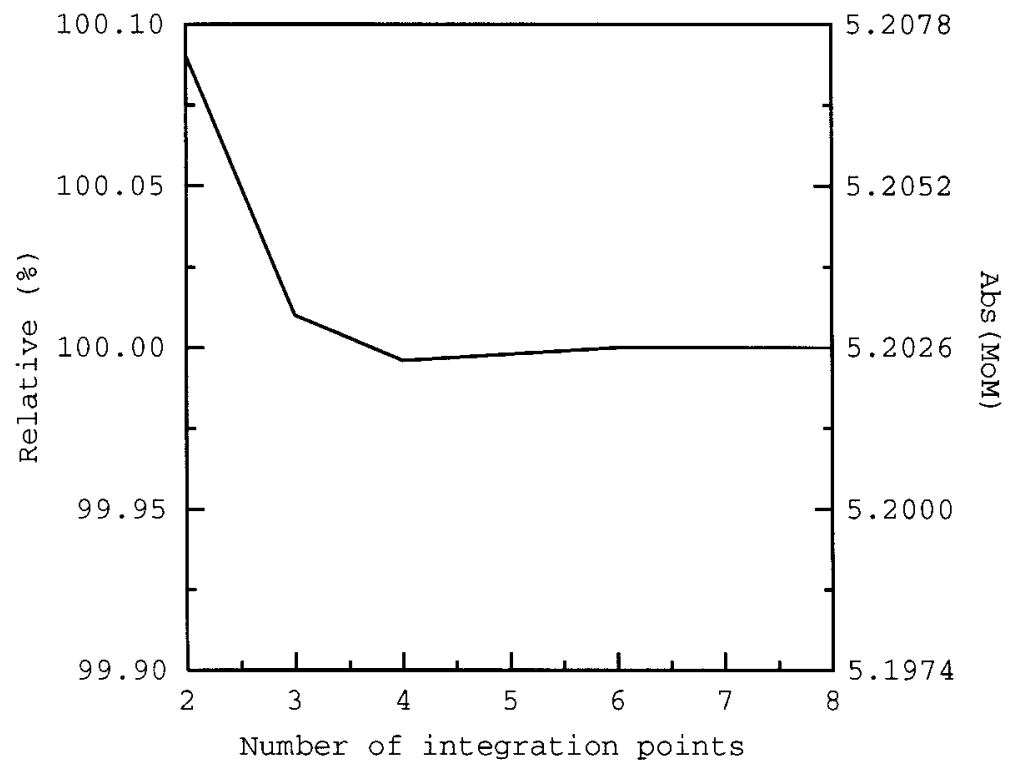

Figure 2 Relative and absolute values of a typical singular MoM element as a function of the number of integration points. Numerical test is performed for the structure shown in Figure 1 and at a frequency of $70 \mathrm{GHz}$

tor and equivalent electric and magnetic surface currents. Physical optics approximation, based on image theory, allows the electric current to be considered as short circuited. The equivalent magnetic surface current is proportional to the electric field on the outer surface of the lens. This field is computed accepting that the lens radius is much larger than the wavelength in the lens dielectric, so that Fresnel's transmission coefficients may be used.

\section{RESULTS}

All simulated and measured results shown below correspond to the integrated antenna/mixer depicted in Figure 1. Figure 3 shows the module of the generalized load reflection coefficient, as defined by Collin [11], in decibels, that is,

$$
\Gamma_{L}=20 \log \left|\frac{Z_{\text {diode }}-Z_{\text {ant }}^{*}}{Z_{\text {diode }}+Z_{\text {ant }}}\right| .
$$

Simulations presented in Figure 3 correspond to two different meshing resolutions. The solid line is obtained when slot and strip widths contain a single cell. To obtain the dotted line, width is divided into three cells. No significant changes are obtained when further increasing the number of transverse cells, which verifies the convergence of the numerical method. In addition, Figure 3 shows the onset of a double-resonance phenomenon, expected from the discussion in Section 1. One resonance is related to the slot loop size, and the other one is determined by the coupling microstrip section length. Bandwidth is then larger than in uniplanar designs. For a reflection coefficient of $-10 \mathrm{~dB}$, the bandwidth is about $20 \%$, which is better than previous designs [7].

Figures 4 and 5 show the simulated and measured radiation patterns in the $E$ - and $H$-planes, respectively, at $65 \mathrm{GHz}$. Figure 6 shows the $E$-plane pattern at $70 \mathrm{GHz}$. The $H$-plane pattern barely changes with frequency, and therefore it is not shown. All of these patterns are for an extension length of $L_{\text {ext }}=8.5 \mathrm{~mm}$, which is between the hyperhemisphere $\left(L_{\text {ext }}=7.2 \mathrm{~mm}\right)$ and the synthesized ellipsoid $\left(L_{\text {ext }} \simeq\right.$ $9.6 \mathrm{~mm})$. Measured patterns show reasonable agreement with

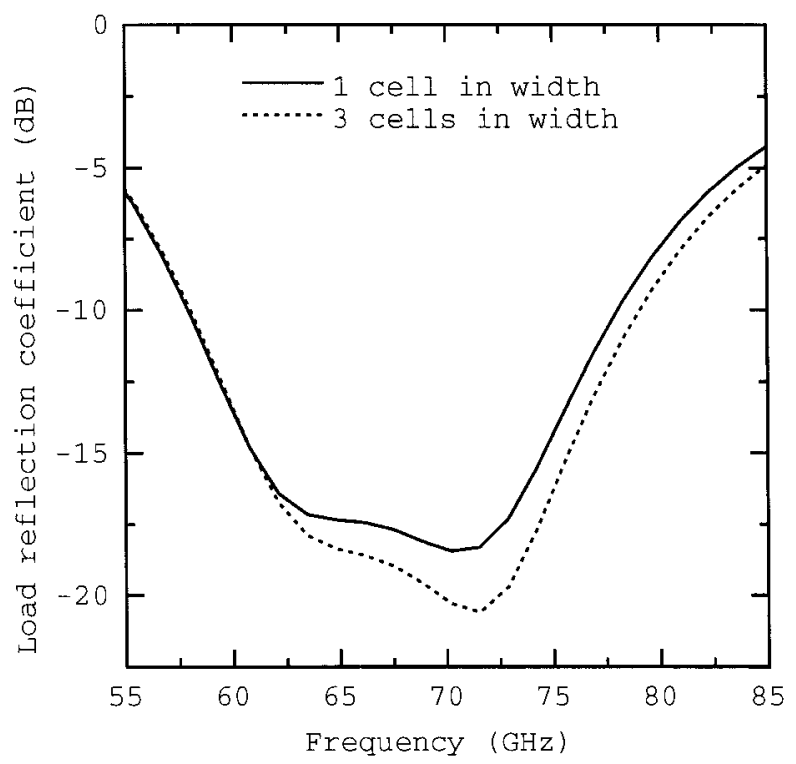

Figure 3 Generalized load reflection coefficient between antenna and diode versus frequency for two different grids

the simulated patterns. Moreover, the $H$-plane pattern shows an extremely low sidelobe level, and it changes very little with frequency. The $E$-plane sidelobe level is not as good as the $H$-plane one. However, it is better than previous designs [2] and improves with frequency, as can be observed in Figure 6. Even though the loop is perfectly symmetric, the equivalent magnetic currents that the microstrip creates in the slot are not uniformly distributed on both sides of the loop because feeding is done only on one side. This fact is considered by our in-house simulation tools, but the predicted asymmetry is noticeably smaller than the measured one, as observed on the left sidelobe of the patterns in Figure 4. The left sidelobe is therefore probably due to uncontrolled spurious propagation phenomena generated by the diode.

Predictions for directivity of the lens antenna at $65 \mathrm{GHz}$ versus extension length are shown in Figure 7. An inflection 


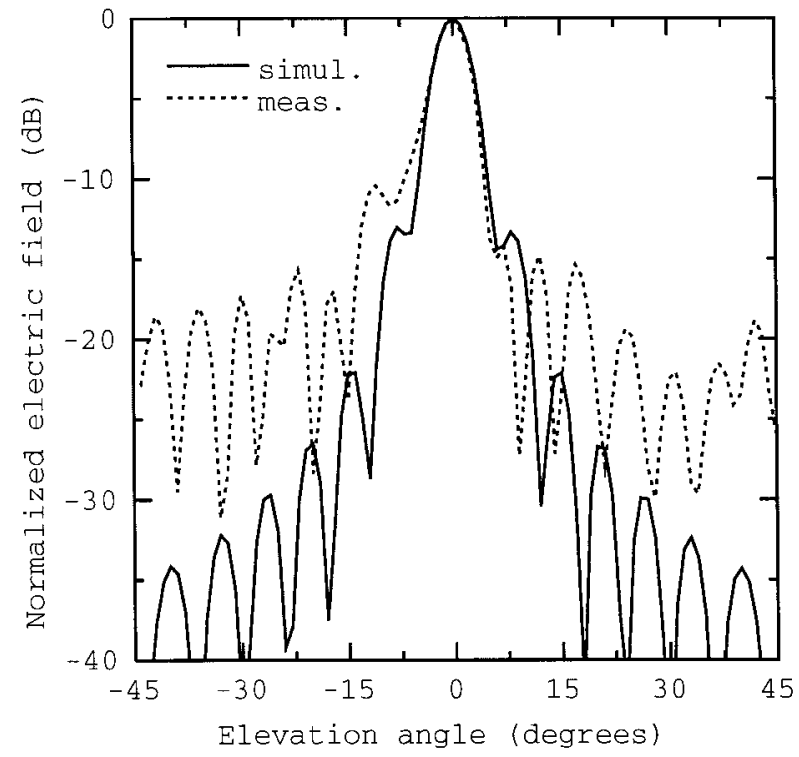

Figure 4 Simulated and measured $E$-plane patterns at $65 \mathrm{GHz}$ with $L_{\text {ext }}=8.5 \mathrm{~mm}$

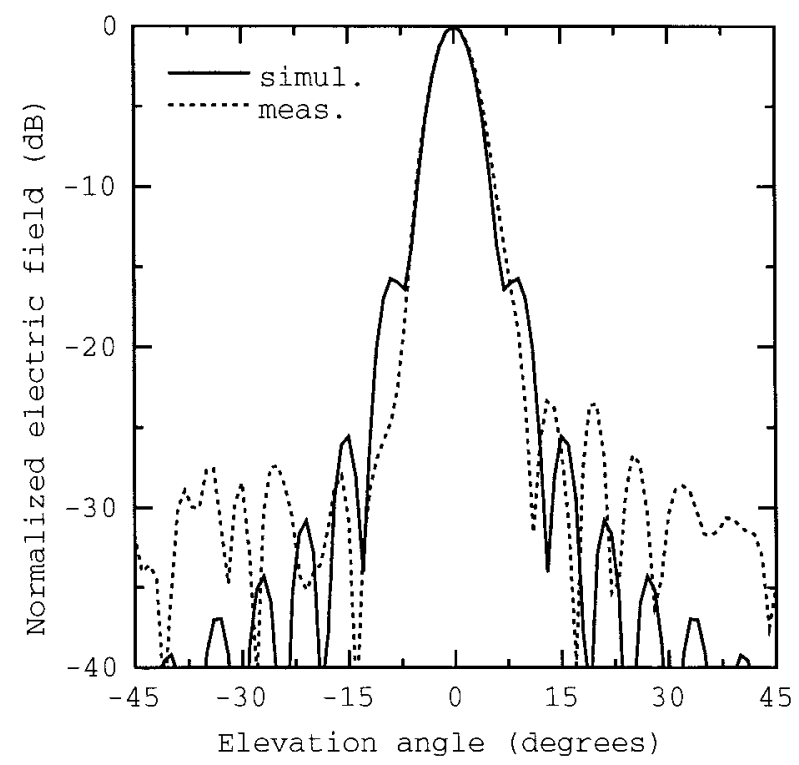

Figure 5 Simulated and measured $H$-plane patterns at $65 \mathrm{GHz}$ with $L_{\text {ext }}=8.5 \mathrm{~mm}$

point is observed at the hyperhemispherical position. The maximum directivity is obtained close to the synthesized ellipsoidal point, as expected. The lens antenna directivity, as well as the input impedance previously shown, cannot be directly measured, so far, at our laboratory. Instead, the isotropic conversion gain (ICG) [12] has been measured, which is shown in Figure 8. The ICG is defined as the power delivered to the IF load from a copolarized incident plane wave by an antenna mixer or an antenna amplifier-mixer, divided by the power that would have been received by an isotropic antenna with a matched load. The ICG is a magnitude that groups antenna and mixer parameters such as the antenna gain (directivity and losses), the antenna/mixing device mismatch, the mixer conversion loss, and the IF filter insertion loss. The ICG is a figure of merit of a frequency converter which may be used when the mentioned parame-

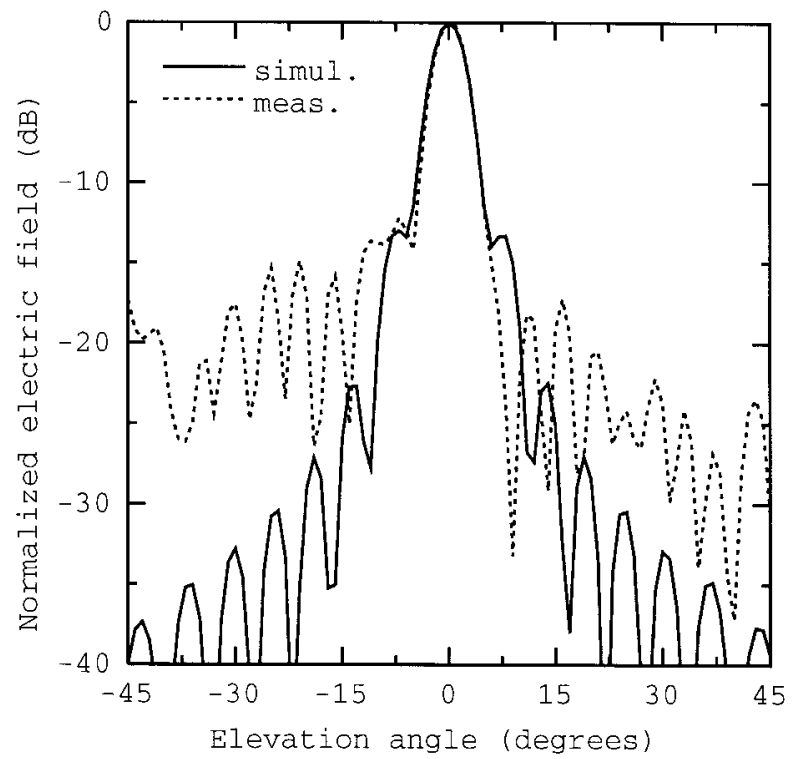

Figure 6 Simulated and measured $E$-plane patterns at $70 \mathrm{GHz}$ with $L_{\text {ext }}=8.5 \mathrm{~mm}$

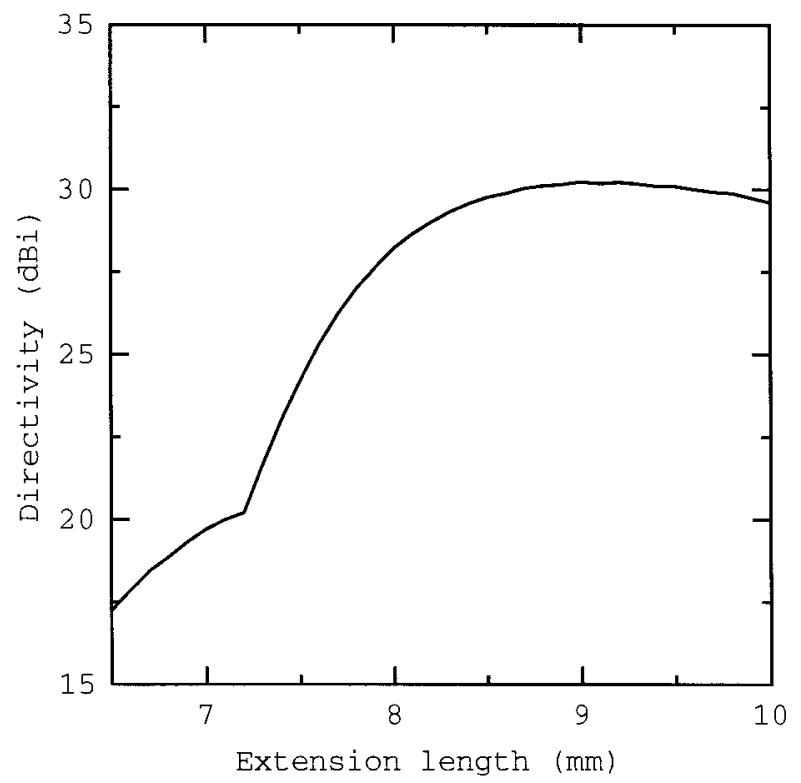

Figure 7 Simulated lens antenna directivity versus extension length at $65 \mathrm{GHz}$

ters cannot be directly measured. In our prototype, the ICG performance is similar to that obtained in previous designs, and good agreement is obtained with predicted bandwidth in Figure 3.

\section{CONCLUSIONS}

A type of square-loop slot antenna, coupled to a microstrip line section, has been examined as a candidate for a millimeter-wave integrated-circuit Schottky-diode mixer. The antenna has been optimized to match the typical input impedance of such diodes in the millimeter-wave band. Optimization has been performed by means of an efficient MoM analysis software tool specifically developed for this study. The antenna shows a very wide frequency band, clean and rotationally symmetric patterns, and a good compromise be- 


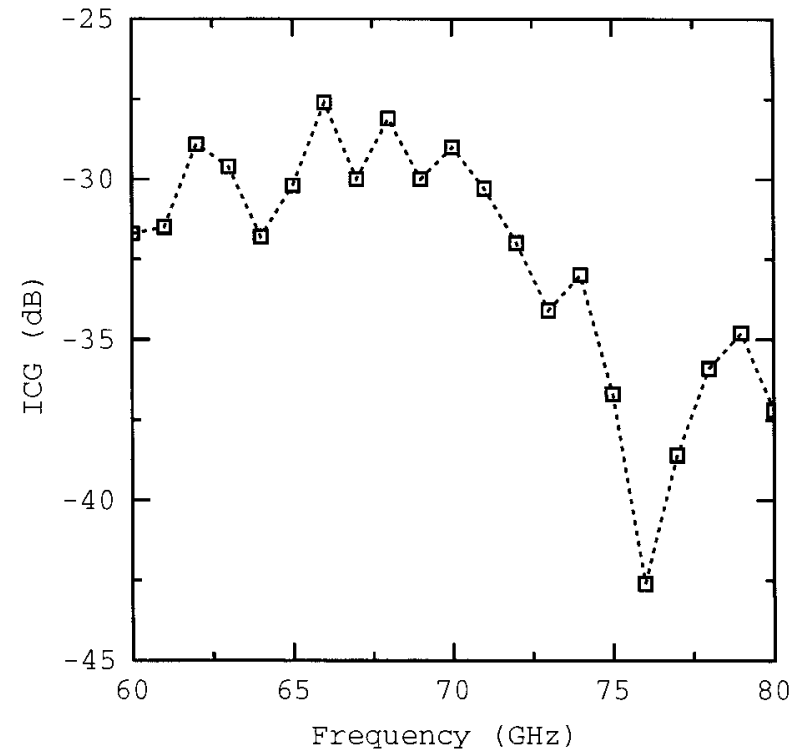

Figure 8 Isotropic conversion gain versus frequency

tween directivity and Gaussian coupling efficiency, depending on the hemispherical lens extension length. Simulation results agree well with measurements.

\section{ACKNOWLEDGMENTS}

The authors are grateful to Prof. F. Gardiol for his encouraging support. Pablo Otero is supported by the Spanish National Science and Technology Agency, CICYT ("Comisión Interministerial de Ciencia y Tecnología").

\section{REFERENCES}

1. D. F. Filipovic, S. S. Gearhart, and G. M. Rebeiz, "Double-Slot Antennas on Extended Hemispherical and Elliptical Silicon Dielectric Lenses," IEEE Trans. Microwave Theory Tech., Oct. 1993, pp. $1738-1749$.

2. P. Otero, G. V. Eleftheriades, and J. R. Mosig, "Slot-Loop Antennas on Substrate Lenses for Submillimeter-Wave Open Structure Mixers," Proc. 20th ESTEC Antenna Workshop, Noordwijk, The Netherlands, June 1997, pp. 193-200.

3. J. P. Daniel, E. Penard, and C. Terret, "Design and Technology of Low-Cost Printed Antennas," in Handbook of Microstrip Antennas, J. R. James and P. S. Hall, Eds., Peter Peregrinus, London, 1989, Vol. 1, Chap. 11, pp. 579-691.

4. Rogers Corporation, 100 South Roosevelt Ave., Chandler, AZ 85226 USA.

5. K. C. Gupta, R. Garg, I. Bahl, and P. Bhartia, Microstrip Lines and Slotlines, 2nd ed., Artech House, Norwood, MA, 1996.

6. D. B. Rutledge, D. P. Neikirk, and D. P. Kasilingam, "Integrated-Circuit Antennas," in Infrared and Millimeter Waves, K. J. Button, Ed., Academic Press, New York, 1983, Vol. 10, Chap. 1, pp. 1-90.

7. P. Otero, G. V. Eleftheriadesand, and J. R. Mosig, "Integrated Modified Rectangular Loop Slot Antenna on Substrate Lenses for $\mathrm{mm}$ - and Submm-Wave Frequencies Mixer Applications," submitted to IEEE Trans. Antennas Propagat., Sept. 1997.

8. J. R. Mosig, "Integral Equation Technique," in Numerical Techniques for Microwave and Millimeter-Wave Passive Structures, T. Itoh, Ed., John Wiley \& Sons, New York, 1989, Chap. 3, pp. 133-213.

9. K. A. Michalski and J. R. Mosig, "Multilayered Media Green's Functions in Integral Equation Formulations," IEEE Trans. Antennas Propagat., Mar. 1997, pp. 508-519.
10. G. V. Eleftheriades, J. F. Zürcher, and J. R. Mosig, "Patterns and Efficiencies of Slot-Fed mm-Wave Glass-Ceramic Substrate Lens Antennas," Proc. ESA / ESTEC Workshop mm-Wave Tech. Appl., Noordwijk, The Netherlands, Nov. 1995, pp. 2.2.1-2.2.13.

11. R. E. Collin, Foundations for Microwave Engineering, 2nd ed., McGraw-Hill, New York, 1992.

12. G. M. Rebeiz, "Millimeter-Wave and Terahertz Integrated Circuit Antennas," Proc. IEEE, Nov. 1992, pp. 1748-1770.

(c) 1998 John Wiley \& Sons, Inc.

CCC 0895-2477/98

\section{THE INPUT IMPEDANCE OF A THIN COAXIAL-LINE-FED PROBE IN A THICK SEMI-INFINITE COAXIAL LINE}

\author{
Z. M. Xie, ${ }^{1}$ Edward K. N. Yung, ${ }^{1}$ and R. S. Chen ${ }^{1}$ \\ ${ }^{1}$ Department of Electronic Engineering \\ City University of Hong Kong \\ Hong Kong
}

Received 5 December 1997

ABSTRACT: The Green's functions for determining the electromagnetic fields in a semi-infinite coaxial line due to a radially directed infinitesimally short electric dipole are derived. The coaxial line is shorted at one end and terminated at a perfectly matched load at the other, and the probe is connected to the inner conductor of this semi-infinite coaxial line and is fed by a smaller coaxial line through a hole on the surface of the outer conductor. Based on these Green's functions, TEM, TE, and TM modes are considered in our analysis, and the input impedance of a small probe in front of the plunger is calculated. As a result, our theoretical results agree very well with the experimental data over a frequency band for several distances between the probe and the plunger. The comparison is also made between our results and those calculated by using traditional transmission line theory, which treats the probe as a transmission line branch. (C) 1998 John Wiley \& Sons, Inc. Microwave Opt Technol Lett 18: 95-100, 1998.

Key words: Green's function; input impedance; coaxial line; mode expansion

\section{INTRODUCTION}

In some applications such as antenna feeding or microwave measurement, the connection must be made between a thick coaxial line and a thin coaxial line. Usually, they are connected longitudinally, which is the case in a coaxial line step. However, when the difference between the diameters of the two coaxial lines is very large, many step impedance transformers are needed to achieve good matching over a frequency band. In this way, the transition has to be long enough, especially for a low RF band. Alternatively, the smaller coaxial line is used as the feeding of a probe connected to the inner conductor of the thick coaxial line, just like a T-junction with one end shorted. The plunger can act as a tuning component. The geometry of the connection is shown in Figure 1. In a low enough frequency, the probe only excites the TEM mode in the large coaxial line, and the T-junction can be viewed as a transmission line branch. Therefore, the input impedance can be calculated by using transmission line theory or a Smith chart. However, as the frequency increases, the effect of a high-order mode excited by the probe cannot be ignored. The above method is not rigorous for engineering design, especially in the case where 